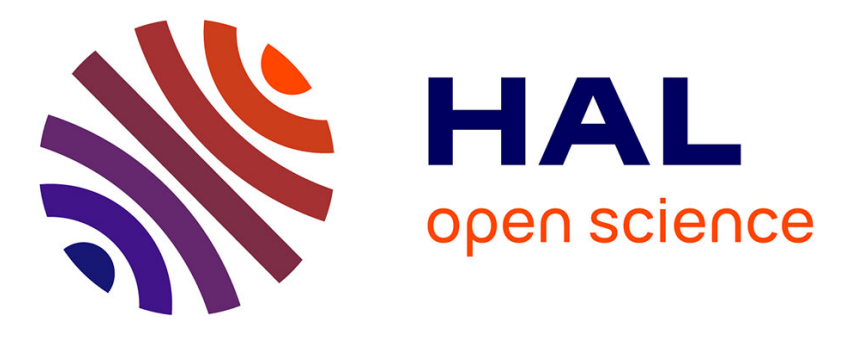

\title{
Variational level-set reconstruction of accretionary morphogenesis from images.
}

Ronan Fablet, Sylvain Pujolle, Anatole Chessel, Abdesslam Benzinou, Frédéric Cao

\section{- To cite this version:}

Ronan Fablet, Sylvain Pujolle, Anatole Chessel, Abdesslam Benzinou, Frédéric Cao. Variational level-set reconstruction of accretionary morphogenesis from images.. ICIP 2006: 13th IEEE International conference on Image Processing, Oct 2006, Atlanta, United States. pp.221 - 224, 10.1109/ICIP.2006.312465 . hal-02344289

\section{HAL Id: hal-02344289 \\ https://hal.science/hal-02344289}

Submitted on 4 Nov 2019

HAL is a multi-disciplinary open access archive for the deposit and dissemination of scientific research documents, whether they are published or not. The documents may come from teaching and research institutions in France or abroad, or from public or private research centers.
L'archive ouverte pluridisciplinaire HAL, est destinée au dépôt et à la diffusion de documents scientifiques de niveau recherche, publiés ou non, émanant des établissements d'enseignement et de recherche français ou étrangers, des laboratoires publics ou privés. 


\title{
VARIATIONAL LEVEL-SET RECONSTRUCTION OF ACCRETIONARY MORPHOGENESIS FROM IMAGES
}

\author{
R. Fablet ${ }^{1}$, S. Pujolle ${ }^{1}$, A. Chessel ${ }^{1}$, A. Benzinou ${ }^{2}$ and F. Cao ${ }^{3}$ \\ Ifremer/LASAA \\ ENIB/RESO \\ IRISA/VISTA \\ BP 70, Technopôle Brest-Iroise \\ Technopôle Brest-Iroise \\ Campus de Beaulieu \\ 29280 Plouzane, FR \\ \{rfablet,achessel\}@ifremer.fr \\ 29280 Plouzane, FR \\ benzinou@enib.fr \\ 35014 Rennes, FR \\ fcao@irisa.fr
}

\begin{abstract}
This paper copes with the reconstruction of accretionary morphogenesis within a given observation plane from an image depicting successive (typically seasonal or daily) growth structures. Modeling accretionary growth shapes as the level-sets of a potential function, a variational framework is derived from geometric criteria. It resorts to minimizing an energy functional involving two terms: a regularization term and a data-driven term which constrain the evolution of the shapes with respect to a growth orientation field. Experiments carried out on real data (e.g., fish otoliths) validate the proposed approach, which opens new research directions for information extraction and decoding from biological archives.
\end{abstract}

\section{INTRODUCTION AND PROBLEM STATEMENT}

Shape deformation has been widely studied in the field of computer vision mainly for applications to shape matching or deformable object analysis $[4,16]$. In this paper, we focus on the deformation of structures which grow according to an accretionary process. In the biological domain, examples of such structures include tree trunks, the shells of shellfish, fish otoliths, corals, bones, vertebrae.... Other examples related to crystallization process could also be cited. The characteristics of the accretionary growth generally vary over time, generally with some given periodicity (mainly daily and annual), in terms of crystalline organization or composition. This leads to the presence of successive dated layers with different optical densities and provides the basis for exploiting such structures as biological archives: for instance, for the reconstruction of the daily temperatures of the environment [6] or for the analysis of individual migration paths [7].

Concerning shape characteristics, the presence of these successive layers potentially permit to back-track the evolution of the shape of the observed structure from the primary core to the outline. The morphogenesis of such structures could then be studied at the individual level, and not only from a statistical point of view at a population level. However, this issue does not reduce to matching successive shapes $[4,16]$,
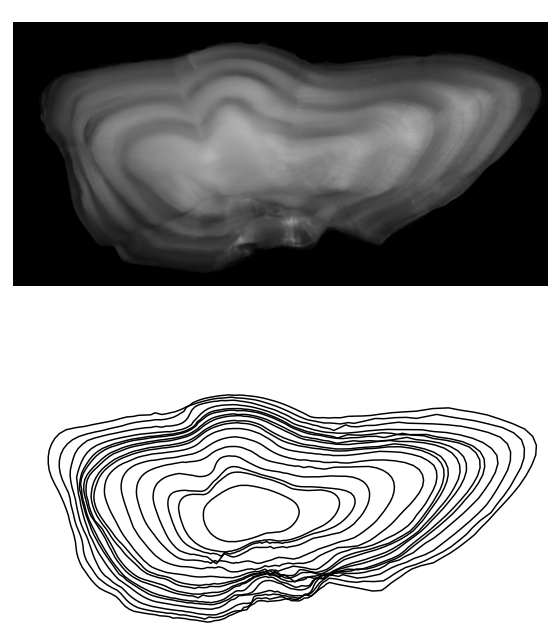

Fig. 1. Illustration of the goal of the paper: image of a structure growing according to an accretionary process (a pollock otolith) in a given observation plane containing the growth center (top); reconstructed series of the evolved shapes (down).

since the automated segmentation of the growth rings is itself a complex problem due to the presence of blind areas and so-called subjective contours [9]. But, it can be stated as an inverse problem for which the goal is to reconstruct the series of the 2D shapes of the observed structure within some observation plane given an image of this structure. Image orientations (i.e., the orientations of image gradients) locally convey relevant information on the shape of the growth rings, since they can be viewed as estimations of the growth directions. Consequently, the reconstruction of the series of shapes of the processed biological structure is solved for such that these shapes are locally normal to image orientations. Formally, a level-set representation of the accretionary growth permits to derive a variational formulation from purely geometric constraints including both orientation-based and shape regularity constraints. The reconstruction of the morphogenesis then re- 


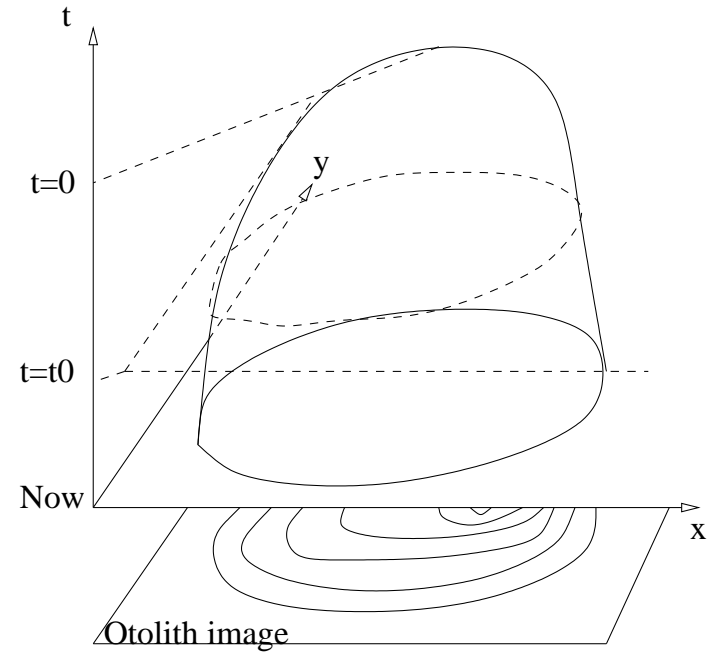

Fig. 2. Level-set representation of the accretionary growth process: the evolution of the shape is described by a potential function $U$, such that the growth shape at time $t$ is its levelline $\Gamma_{t}(U)=\left\{p \in \mathcal{R}^{2}\right.$ such that $\left.U(p)=t\right\}$.

sorts to a variational interpolation which is efficiently solved for within a multiresolution framework.

\section{PROPOSED APPROACH}

\subsection{Joint level-set representation and variational setting}

The accretionary growth process is modeled as a potential function $U$ defined over $\mathcal{R}^{2}$ with values in $[O, T]$ such that the level set $\Gamma_{t}(U)$ of $U$ for level $t$ is the shape of the considered biological structure within a given observation plane at time $t$, that is to say

$$
\Gamma_{t}(U)=\left\{p \in \mathcal{R}^{2} \text { such that } U(p)=t\right\}
$$

Intrinsically, such a representation guarantees that the growth is normal to the shape, as commonly assumed for accretionary growth. Note that, in practice, the actual tile information might not be available, so that the values of potential function $U$ are not actual time values, but are homogeneous to time.

Based on this level-set representation, we aim at estimating the potential function $U$ associated with a given biological structure. Formally, some boundary conditions are assumed to be provided. These conditions include the shapes at times 0 and $\mathrm{T}$, which may be automatically extracted [3]. solving for the reconstruction of $U$ is then stated as the computation of the extension of the boundary values $G_{\mathcal{B}}$ associated with boundary conditions $\mathcal{B}$, to the whole domain $\mathcal{R}^{2}$ according to some energy criterion $E$ :

$$
\widehat{U}=\arg \min _{U \in \mathcal{U}\left(G_{\mathcal{B}}\right)} E(U),
$$

where $\mathcal{U}\left(G_{\mathcal{B}}\right)$ is the set of the functions from $\mathcal{R}^{2}$ to $\mathcal{R}$ which are equal to $G_{\mathcal{B}}$ on boundary set $\mathcal{B}$. Energy $E(U)$ is split into two terms: a regularization term issued from a geometric shape prior and a data-driven term setting orientation-based constraints:

$$
E(U)=E_{R}(U)+\gamma E_{O}(U),
$$

where $\gamma$ is a weighting factor balancing the relative influence of each term. The regularization term is defined as the sum of the perimeter of the growth shapes computed as

$$
E_{R}(U)=\int_{t \in[0, T]} \int_{p \in \Gamma_{t}(U)} 1
$$

The second term relies on orientation-based constraints. More precisely, the gradients of the image depicting the observed biological structure permit to locally infer the local growth orientations. Let us denote by $\omega$ the orientation field computed as the normalized image gradient $\nabla I /|\nabla I|$, where $\nabla I$ is the gradient of image $I$. For low gradient values, the computation of the orientation is highly noisy. The norm of the gradient is however known as a relevant measure of the reliability of this gradient information. Thus, a confidence map $\alpha$ is defined as $g(|\nabla I|)$, where $g$ is a continuous stepwise function rescaled between 0 and 1 . As the normal to shape $\Gamma_{t}(U)$ is expected to be tangent to orientation $\omega$ given the confidence weight $\alpha$, energy term $E_{O}(U)$ is computed as follows

$$
E_{O}(U)=\int_{t \in[0, T]} \int_{p \in \Gamma_{t}(U)} \alpha(p) \cdot\left\langle\frac{\nabla F(p)}{|\nabla U(p)|}, \omega(p)^{\perp}\right\rangle^{2}
$$

Minimizing explicitly criterion $E(U)$ is however infeasible in practice, since it would require to extract all the levellines of $U$. From the co-area formula [12], an equivalent formulation can be derived such that the resulting criterion only involves the spatial derivatives of $U$. More precisely, the minimization of $E(U)$ resorts to:

$$
\widehat{U}=\arg \min _{U \in \mathcal{U}\left(G_{\mathcal{B}}\right)} \int_{p \in \mathcal{R}^{2}}|\nabla U|+\gamma \cdot \alpha \cdot|\nabla U| \cdot \rho\left(\left\langle\frac{\nabla U}{|\nabla U|}, \omega^{\perp}\right\rangle\right)
$$

This variational setting involves two classical terms: the first one is regularization term set as a the norm of the gradient of the potential function and the second is the data-driven term, which aims at aligning the orientation field of $U$ to orientation constraints $\omega$. In this second term, norm $|\nabla U|$, inherited from the co-area transform, is a weighting factor such that more influence is given to points where the gradient of the level-set representation is high (conversely, where the growth is slow).

Criterion (6) is derived from purely geometric constraints. In addition, level-set representations are contrast invariant (i.e., a contrast change does not affect the geometry of the levelsets but only leads to different indexes for each level-set) [13]. Consequently, additional constraints are required to prevent 
from retrieving unstable solutions. As constraints on the surface between successive level-lines are equivalent to constraints on first-order statistics $p(U)$ of $U$, minimization (6) will be carried out subject to $p(U)$ is uniform. The choice of a uniform prior is arbitrary. Other priors might be used, if available, for instance priors related to a priori growth laws.

\subsection{Minimization issue}

To carry out the above constrained minimization, a two-step iterative approach is proposed: the first step solves for unconstrained minimization (6), and the second step carries out an histogram equalization [10] to map the current solution onto the constraint that $p(U)$ is uniform. We detail below the unconstrained minimization. Besides, to improve the convergence of this procedure and reduce the dependence with respect to the initialization, we adopt a multiresolution framework.

Unconstrained minimization (6) is solved for using a discrete/Markovian setting of the associated variational criterion. Hence, energy $E(U=$ is computed as:

$$
\sum_{p \in \mathcal{R}^{2}}\left|\nabla U_{p}\right|+\gamma \cdot \alpha_{p} \cdot\left|\nabla U_{p}\right| \cdot \rho\left(\left\langle\frac{\nabla U_{p}}{\left|\nabla U_{p}\right|}, \omega_{p}^{\perp}\right\rangle\right),
$$

where $\nabla U_{p}$ is approximated as $\left(U_{p_{1}}-U_{p_{3}}, U_{p_{2}}-U_{p_{4}}\right)$ with $p_{1}, p_{2}, p_{3}, p_{4}$ the four western, northern, eastern and southern neighbors of pixel $p$ in the image. Besides, as the absolute norm || is not derivable in 0 , its use may lead to numerical instabilities. Therefore, it is replaced by $\rho(x)=\sqrt{\epsilon^{2}+x^{2}}$. An iterated weighted least square (IRLS) scheme is then exploited to minimize the resulting criterion [11]. This schemes iterates the computation of weights $\beta(p)=\phi\left(\nabla U_{p}\right)$, where $\phi(x)=\rho^{\prime}(x) / x$, and the computation of the solution of the weighted least-square issue

$$
\min _{U \in \mathcal{U}\left(G_{\mathcal{B}}\right)} \sum_{p \in \mathcal{R}^{2}} \beta(p)\left|\nabla U_{p}\right|^{2}+\gamma \cdot \alpha_{p} \cdot\left\langle\frac{\nabla U_{p}}{\sqrt{\left|\nabla U_{p}\right|}}, \omega_{p}^{\perp}\right\rangle^{2}
$$

given weight maps $\beta$. Using an incremental Gauss-Seidel scheme, we recursively solve for the optimal incremental update of potential $U_{p}$ at pixel $p$ given the potential function elsewhere. This step relies on a linearization of the cost function with respect to the increment. This incremental refinement is applied to all pixels except those belonging to boundary set $\mathcal{B}$.

From the updated solution $\tilde{U}$ of the unconstrained criterion (6), a new solution is derived such that the constraint that $p(U)$ is uniform applies. This step simply comes to implementing an equalization of the histogram of $\tilde{U}$. As stressed previously, this equalization step only changes the contrast between level-lines, but the geometry of the level-set representation remains unchanged. For computational efficiency, this equalization step is not applied after each incremental update, but typically every one hundred iterations. This parameter setting was experimentally proven to ensure the convergence to non-degenerate estimations.

\subsection{Multiresolution minimization}

Minimization (6) is obviously not convex. To prevent from being trapped in local minima and to ensure a better robustness to the initialization, a multiresolution scheme is used. Given a Gaussian pyramid of the orientation field $\omega$, the multiresolution approach comes to solve for the estimation of the potential function $U$ at successive resolutions, from the coarsest resolution to the finest one. The final estimate at a given resolution serves as the initialization at the next resolution. In practice, Gaussian pyramids with four levels are exploited.

\subsection{Initialization}

The computation of a relevant initialization to the proposed gradient-based multiresolution scheme is important to ensure a fast and pertinent convergence. The initialization is delivered by the AMLE (Absolutely Minimizing Lipschitz Extension) of the boundary conditions to the whole domain [5]. Since the AMLE resorts to the minimization of the $L_{\infty}$ norm, the AMLE can be viewed as the less smooth interpolation operator among all interpolation operator. The AMLE is implemented as a diffusion operator $\partial U / d t=U_{\nabla \nabla}$ where $U_{\nabla \nabla}$ is the second-order derivative in the direction of the gradient of $U$. The key properties of the AMLE is that it is an artifactfree and oscillation-free interpolant actually capable of taking into account isolated boundary points as well as curves.

\section{RESULTS}

Results of the reconstruction of the morphogenesis of different real structures are reported to demonstrate the efficiency of the proposed method. In all these results the boundary conditions are simply issued from the extraction of the outline and the position of the manually marked growth center. The parameter setting was defined as follows: potential values of $\mathrm{U}$ ranging from $\mathrm{O}$ (growth center) to 1 (outline), $\gamma=10$ and $\epsilon=1$. Concerning computational time, the proposed method runs in about one minute for $1000 \times 1000$ images under a Pentium IV at $2.5 \mathrm{GHz}$.

Fig.3 reports a first example with a pollock otolith. Animated versions of these results can be viewed at webpage www.ifremer.fr/lasaa/rfablet/mottolith. The observed rings are seasonal rings (dark zones are winter rings whereas white zones correspond to summer rings). It should however be noted that none of the annual rings is actually closed. They all involve subjective contours to be closed (especially the first dark ring). Consequently, extracting closed rings appear as complex issue if no additional a priori information is available. Conversely, the proposed framework successfully re- 

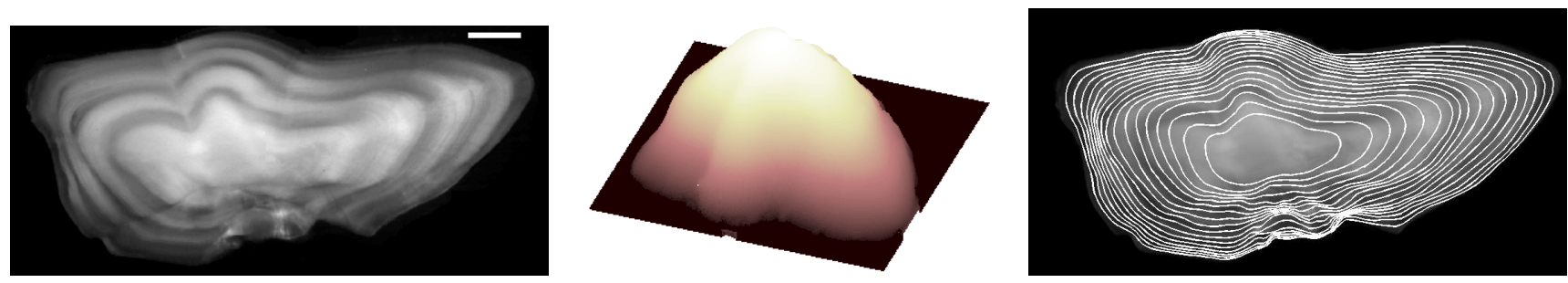

Fig. 3. Reconstruction of the series of growth shapes for the pollock (Pollachius pollachius) otolith image depicted above based on the gradient-based orientation field: image of the pollock otolith (left), reconstructed potential function $U$ (middle), series of shape superposed to the otolith image (right).
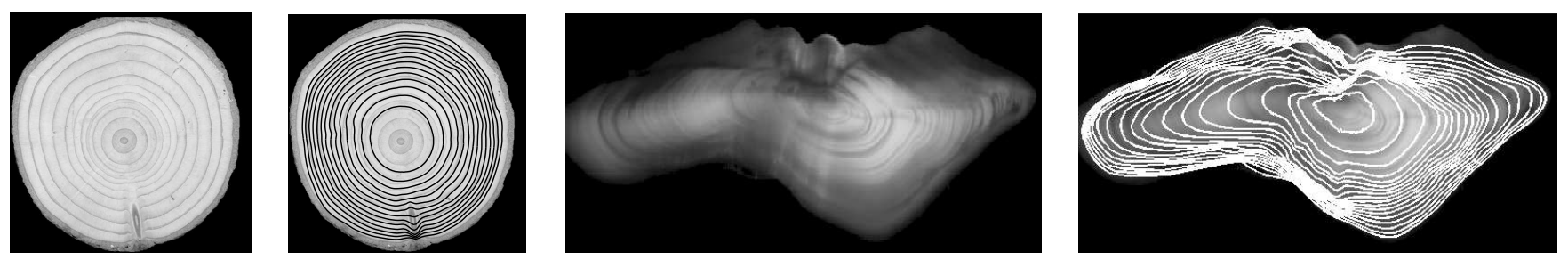

Fig. 4. Examples of reconstruction of the series of growth shapes: image of the tree trunk section (left), reconstructed potential function $U$ (middle), series of shape superposed to the processed image (middle left), image of a hake otolith section (left), reconstructed potential function $U$ (middle right), series of shape superposed to the processed image (right).

cover the deformations of the otolith from the core to the outline. The proposed level-set representation intrinsically encodes that closed shapes have to be reconstructed. Besides, the constraints issued from local image orientations convey sufficient information so that the estimated level-set representation approximately fits to the observed growth rings. Note, for instance, that local shape deformations such as the one occurring towards the top of the otolith is correctly taken into account. Besides, it can stressed that the left and right otolith sides are correctly matched. Results reported in Fig. further illustrates the relevance of the proposed framework for a tree trunk involving rather circular concentric ring shapes, and a hake otolith involving complex ring structures. In both cases, relevant shape series are reconstructed regarding the complexity of the task.

The proposed solution for the reconstruction of the accretionary morphogenesis of biological structures open new research directions with a view to exploiting the invaluable potential of these biological archives in terms of information extraction (growth axis extraction, ring segmentation), morphogenesis analysis and modeling as well as 2D information calibration and decoding (for instance, to analyze information along different growth axis).

\section{REFERENCES}

[1] S. Campana and J. Casselman. Stock discrimination using otolith shape analysis. Can. Jal of Fish. Res. and Aqu. Sc., 50:1062-1083, 1993.

[2] F. Cao and R. Fablet. Automatic morphological detection of otolith nucleus. Pattern Recognition Letters, 2006.
[3] V. Caselles, R. Kimmel, and G. Sapiro. Geodesic active contours. International Journal of Computer Vision, 22(1):61-79, 1997.

[4] V. Caselles, J.-M. Morel, and C. Sbert. An axiomatic approach to image interpolation. IEEE Trans. on Image Processing, 7:376-386, 1998.

[5] L. Chauvaud. The shell of the Great Scallop Pecten maximus as a tool for high frequency paleoenvironmental reconstruction. Geochemistry, Geophysics, Geosystems 6, 2004. In press.

[6] F. Daverat and R. Fablet. Reconstruction of life histories chronologies from measures sampled on the otolith using mixture models and hidden Markov models: case study of the eel (anguilla anguilla) otolith $\mathrm{Sr}: \mathrm{Ca}$ ratios. Can. Jal on Fish. and Aq. Sc., 2006. Submitted.

[7] S. Durand and J. Froment. Reconstruction of wavelet coefficients using total variation minimization. SIAM Jal Scientific Computing, 24(5):1754-1767, 2003.

[8] R. Fablet. Semi-local extraction of ring structures in images of biological hard tissues: application to the bayesian interpretation of fish otoliths for age and growth estimation. Can. Jal on Fish. and Aq. Sc., 2006. To appear.

[9] R. Gonzalez and R. Woods. Digital Image Processing. Addison-Wesley Publishing Company, 1992.

[10] P. Huber. Robust Statistics. John Wiley \& Sons, 1981.

[11] F. Lin and X. Yang. Geometric Measure Theory: An Introduction. International Press, 2002.

[12] P. Monasse and F. Guichard. Fast computation of a contrast invariant representation. IEEE Trans. on Image Processing, 9(5):860-872, 2000.

[13] J.M. Odobez and P. Bouthemy. Separation of moving regions from background in an image sequence acquired with a mobile camera. In Video Data Compression for Multimedia Computing, chapter 8, pages 295-311. H. H. Li, S. Sun, and H. Derin, eds, Kluwer, 1997.

[14] L. Rudin, S. Osher, and E. Fatemi. Nonlinear total variation based noise removal algorithms. Physica D, 60(1-4):259-268, 1992.

[15] L. Younès. Optimal matching between shapes via elastic deformations. Image and Vision Computing, 17:381-389, 1999. 